\title{
Research on single carrier frequency domain equalization system based on FQPSK modulation
}

\author{
Meng Zhang ${ }^{1, *}$ and Zhisong Bie ${ }^{1, \mathrm{a}}$ \\ ${ }^{1}$ Beijing University of Posts and Telecommunications, Xitucheng Road 10\#, Haidian District, Beijing, \\ P.R.China. \\ ${ }^{2}$ zhisongbie@bupt.edu.cn
}

\begin{abstract}
Feher-patented quadrature phase shift keying (fqpsk) modulation is proposed for single-carrier frequency domain equalization (sc-fde). this paper puts forward a new method modulation called cc-bcd-fqpsk. the fqpsk signal is oversampled to make that the discrete fourier transform (dft)/ inverse dft (idft) processing of it can be applied for sc-fde. the methodology for maintaining waveform continuity with a cyclic prefix (cp) is presented. wrap-around viterbi algorithm (wava) is suggested for demodulation after equalization at the receiver. simulation results show that sc-fde system based on cc-bcd-fqpsk modulation has higher spectral efficiency than linear modulation qpsk and is an effective approach for eliminating isi and using nonlinear high power amplifier.
\end{abstract}

\section{Introduction}

In recent years, high-speed data communication system such as aircraft-to-earth communications have been developing continuously, and the transmission of high-speed data has become the focus of research. The wireless channel environment changes at any time and is very complex, and multipath fading through wireless channel leads to frequency selective fading and inter symbol interference (ISI). As a kind of equalization technology to effectively eliminate ISI, single carrier frequency domain equalization (SC-FDE) has lower peak to average ratio (PAPR) and sensitivity to frequency offset, which makes it more popular than orthogonal frequency division multiplexing (OFDM) in the case of using high power nonlinear power amplifier.

At present, some linear modulation schemes have been applied to SC-FDE, but one of the obvious drawbacks of linear modulation is that the envelope jitter of the signal is very serious and very sensitive to nonlinear distortion, which leads to high requirement for power amplifier. The aircraft-to-earth communication system often requires high power efficiency, so efficient nonlinear modulation is considered to solve this drawback.

FQPSK was first proposed by Kato and Feher in 1983 and belongs to IJF-OQPSK [3, 4]. FQPSK signal has quasi constant envelope characteristics, which is insensitive to nonlinear

\footnotetext{
* Corresponding author: zhangmeng4113@163.com
} 
distortion and can be used in nonlinear power amplifiers without considering the power back-off problem to achieve high power efficiency. At the same time, the FQPSK signal is continuous without mutation, which makes the FQPSK signal spectrum more compact, less high frequency components, and higher frequency band utilization. Due to its excellent spectral efficiency, power efficiency and low bit error rate, FQPSK has received extensive attention in the field of deep space communications and space telemetry.

Therefore, this paper studies the SC-FDE system based on FQPSK modulation, so that the high-speed data communication system such as aircraft-to-earth communications can not only achieve low complexity against ISI in the frequency domain, but also can play the spectrum advantages of FQPSK to attain higher power efficiency. But there are two issues need to be considered. Firstly, FQPSK signal is changing continuously, so discrete Fourier transform (DFT)/ inverse DFT (IDFT) cannot be directly applied to the received samples. Secondly, FQPSK signal needs to be added a cyclic prefix, because it cannot be directly used for SC-FDE as a non-linear modulation. When the cyclic prefix is added, the phase continuity of the signal needs to be maintained. Hence, this paper discusses how to add a cyclic prefix for applying FQPSK modulation to SC-FDE system.

The remainder of this paper is as follow. Section 2 reviews FQPSK modulation and introduces a new CC-BCD-FQPSK modulation. Section 3 constructs the SC-FDE system based on CC-BCD-FQPSK modulation. Section 4 gives the performance simulation results over the spatial channel model (SCM) channel.

\section{CC-BCD-FQPSK modulation}

A block diagram of FQPSK modulator is shown as follow Figure 1, where a crosscorrelator unit is added after the IJF encoder unit, which reduces the 3-dB envelop fluctuation of IJF-FQPSK nearly to 0 -dB to make the signal appears constant envelope.

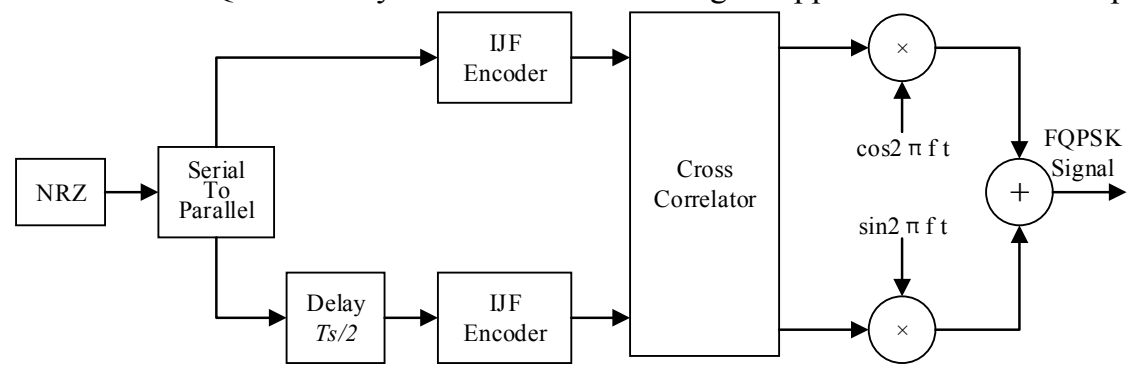

Fig. 1. The diagram of FQPSK modulation

Simon, M.K. and Yan, T.Y. proposed a symbol-by-symbol cross-correlator mapping method for FQPSK and implemented it with trellis code [3]. Define 16 basic waveforms, $s_{i}(t) ;-T_{s} / 2 \leq t \leq T_{s} / 2 ; i=0,1,2, \cdots, 15$ as Eq. (1), which together form the transmitted signal set of the I and Q path. 


$$
\begin{aligned}
& s_{0}(t)=A,-\frac{T_{s}}{2} \leq t \leq \frac{T_{s}}{2}, \quad s_{8}(t)=-s_{0}(t) \\
& s_{1}(t)=\left\{\begin{array}{cl}
A, & -\frac{T_{s}}{2} \leq t \leq 0 \\
1-(1-A) \cos ^{2} \frac{\pi t}{T_{s}}, & 0 \leq t \leq \frac{T_{s}}{2}
\end{array} \quad s_{9}(t)=-s_{1}(t)\right. \\
& s_{2}(t)=\left\{\begin{array}{crr}
1-(1-A) \cos ^{2} \frac{\pi t}{T_{s}}, & -\frac{T_{s}}{2} \leq t \leq 0 \\
A, & 0 \leq t \leq \frac{T_{s}}{2} &
\end{array}\right. \\
& s_{3}(t)=1-(1-A) \cos ^{2} \frac{\pi t}{T_{s}},-\frac{T_{s}}{2} \leq t \leq \frac{T_{s}}{2} \quad s_{11}(t)=-s_{3}(t) \\
& s_{4}(t)=A \sin \frac{\pi t}{T_{s}},-\frac{T_{s}}{2} \leq t \leq \frac{T_{s}}{2} \quad s_{12}(t)=-s_{4}(t) \\
& S_{5}(t)=\left\{\begin{array}{l}
\sin \frac{\pi t}{T_{s}}+(1-A) \sin ^{2} \frac{\pi t}{T_{s}}, \quad-\frac{T_{s}}{2} \leq t \leq 0 \\
\sin \frac{\pi t}{T_{s}}, \quad 0 \leq t \leq \frac{T_{s}}{2}
\end{array} \quad S_{13}(t)=-S_{5}(t)\right. \\
& s_{6}(t)=\left\{\begin{array}{l}
\sin \frac{\pi t}{T_{s}}, \quad-\frac{T_{s}}{2} \leq t \leq 0 \\
\sin \frac{\pi t}{T_{s}}-(1-A) \sin ^{2} \frac{\pi t}{T_{s}}, \quad 0 \leq t \leq \frac{T_{s}}{2}
\end{array} \quad S_{14}(t)=-s_{6}(t)\right. \\
& s_{7}(t)=\sin \frac{\pi t}{T_{s}},-\frac{T_{s}}{2} \leq t \leq \frac{T_{s}}{2} \quad s_{15}(t)=-s_{7}(t)
\end{aligned}
$$

All 16 waveforms have continuous slope throughout the defining intervals, and have zero slope at the end points, so that the FQPSK signal has no slope discontinuity anywhere regardless of the value of $A$. The waveforms are shown in Figure 2, and the envelope fluctuation of the FQPSK signal is $0 \mathrm{~dB}$ in the case $A=1 / \sqrt{2}$.

Define $d_{I n}, d_{Q n}$ as I, Q path data symbols respectively in the interval $\left[(n-[1 / 2]) T_{s},(n+[1 / 2]) T_{s}\right]$. And the corresponding mapping symbols $D_{I n}, D_{Q n}$, which both range over the set $(0,1)$, are defined as follow Eq. (2).

$$
\left.\begin{array}{l}
D_{I n} \triangleq \frac{1-d_{I n}}{2} \\
D_{Q_{n}} \triangleq \frac{1-d_{Q_{n}}}{2}
\end{array}\right\}
$$

Then, define the binary-coded decimal (BCD) representation of the indices $i, j$ by

$$
\left.\begin{array}{l}
i=I_{3} \times 2^{3}+I_{2} \times 2^{2}+I_{1} \times 2^{1}+I_{0} \times 2^{0} \\
j=Q_{3} \times 2^{3}+Q_{2} \times 2^{2}+Q_{1} \times 2^{1}+Q_{0} \times 2^{0}
\end{array}\right\}
$$


with

$$
\left.\begin{array}{ll}
I_{0}=D_{Q n} \oplus D_{Q, n-1}, & Q_{0}=D_{I, n+1} \oplus D_{I n} \\
I_{1}=D_{Q, n-1} \oplus D_{Q, n-2}, & Q_{1}=D_{I n} \oplus D_{I, n-1}=I_{2} \\
I_{2}=D_{I n} \oplus D_{I, n-1}, & Q_{2}=D_{Q n} \oplus D_{Q, n-1}=I_{0} \\
I_{3}=D_{I n}, & Q_{3}=D_{Q n}
\end{array}\right\}
$$
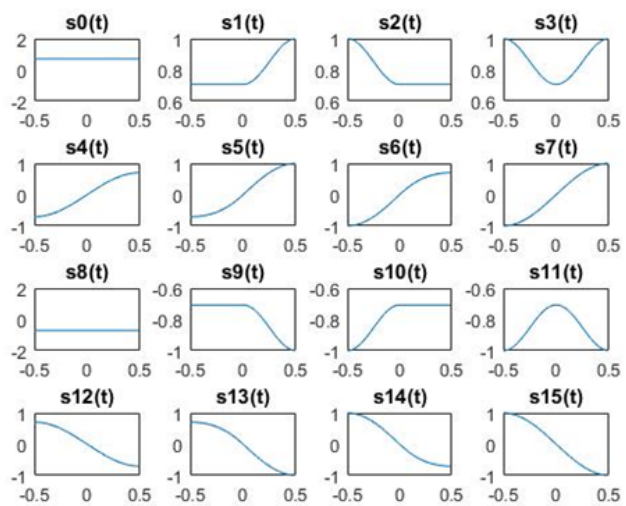

Fig. 2. 16 basic waveforms ( $T_{s}=1, A=1 / \sqrt{2}$ )

The implementation of FQPSK modulation via convolutional code cascaded with binary-coded decimal (CC-BCD) is shown as Figure 3, which can be recognized as a twoparallel-level convolutional encoder cascaded with a BCD mapping unit. The convolutional encoder $\left(6,2,\left[\begin{array}{ll}3 & 3\end{array}\right]\right)$ has two parallel levels with 2 inputs $D_{I n}, D_{Q n}$ and 6 outputs $I_{3}$, $I_{2}=Q_{1}, Q_{0}, Q_{3}, I_{1}, I_{0}=Q_{2}$, and each level has 2 shift registers. The outputs of the convolutional encoder are mapped by the BCD mapping unit to generate $i$ and $j$, which are the indices used to select waveforms from the 16 basic waveforms to form the transmitted signals of the I, Q path at the corresponding time.

$$
\begin{aligned}
& D_{I, n+1} \quad D_{I n} \quad D_{I, n-1} \\
& s_{I}(t)=s_{i}(t)
\end{aligned}
$$

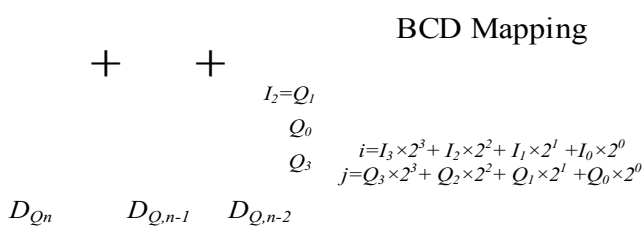

$$
\begin{aligned}
& +\quad+\underset{\substack{I_{1} \\
I_{0}=Q_{2}}}{ }
\end{aligned}
$$

Fig. 3. The diagram of CC-BCD-FQPSK modulation 
The state transitions and outputs of CC-BCD code mapping are shown as Table 1. The first row indicates current two-level registers state $\left(D_{Q, n-1} D_{Q, n-2} D_{I n} D_{I, n-1}\right)$, the first column indicates current input $\left(D_{I, n+1} D_{Q n}\right)$, and the results indicate next state of the convolutional encoder and indices $(i, j)$ of I and Q paths transmitted signals outputted by BCD mapping under current state and current input.

Table 1. State transitions and outputs of CC-BCD code mapping

\begin{tabular}{|c|c|c|c|c|}
\hline & 0 & 1 & 2 & 3 \\
\hline 0 & $0 /(0,0)$ & $8 /(1,12)$ & $2 /(0,1)$ & $10 /(1,13)$ \\
\hline 1 & $0 /(4,2)$ & $8 /(5,14)$ & $2 /(4,3)$ & $10 /(5,15)$ \\
\hline 2 & $1 /(12,3)$ & $9 /(13,15)$ & $3 /(12,2)$ & $11 /(13,14)$ \\
\hline 3 & $1 /(8,1)$ & $9 /(9,13)$ & $3 /(8,0)$ & $11 /(9,12)$ \\
\hline 4 & $0 /(2,0)$ & $8 /(3,12)$ & $2 /(2,1)$ & $10 /(3,13)$ \\
\hline 5 & $0 /(6,2)$ & $8 /(7,14)$ & $2 /(6,3)$ & $10 /(7,15)$ \\
\hline 6 & $1 /(14,3)$ & $9 /(15,15)$ & $3 /(14,2)$ & $11 /(15,14)$ \\
\hline 7 & $1 /(10,1)$ & $9 /(11,13)$ & $3 /(10,0)$ & $11 /(11,12)$ \\
\hline 8 & $4 /(3,4)$ & $12 /(2,8)$ & $6 /(3,5)$ & $14 /(2,9)$ \\
\hline 9 & $4 /(7,6)$ & $12 /(6,10)$ & $6 /(7,7)$ & $14 /(6,11)$ \\
\hline 10 & $5 /(15,7)$ & $13 /(14,11)$ & $7 /(15,6)$ & $15 /(14,10)$ \\
\hline 11 & $5 /(11,5)$ & $13 /(10,9)$ & $7 /(11,4)$ & $15 /(10,8)$ \\
\hline 12 & $4 /(1,4)$ & $12 /(0,8)$ & $6 /(1,5)$ & $14 /(0,9)$ \\
\hline 13 & $4 /(5,6)$ & $12 /(4,10)$ & $6 /(5,7)$ & $14 /(4,11)$ \\
\hline 14 & $5 /(13,7)$ & $13 /(12,11)$ & $7 /(13,6)$ & $15 /(12,10)$ \\
\hline 15 & $5 /(9,5)$ & $13 /(8,9)$ & $7 /(9,4)$ & $15 /(8,8)$ \\
\hline
\end{tabular}

\section{SC-FDE system based on CC-BCD-FQPSK modulation}

As mentioned in the introduction, there aretwo problems to consider when applying FQPSK signal to SC-FDE. According to section 2, the CC-BCD-FQPSK signal is composed of the 16 basic waveforms, which are all continuous in the interval $-T_{s} / 2 \leq t \leq T_{s} / 2$ without mutation point, so the transmitted signal is continuous. To ensure that the received samples can be applied with DFT/IDFTand fully recovered, the CC-BCD-FQPSK signal needs to be oversampledat the transmitter, so that the discrete CC-BCD-FQPSK signal can be applied for SC-FDE.

Therefore, the SC-FDE system model based on CC-BCD-FQPSK modulation is built and illustrated as Figure 4, which involves cyclic prefix design, frequency domain equalization, and demodulation and so on. The binary source inputs are modulated by CCBCD-FQPSK modulation after adding cyclic prefix, then sent to channel. At the receiver, after the received signal is synchronized, cyclic prefix is removed, block DFT is carried out, and equalization is carried out in the frequency domain, the signal is converted back to the time domain for demodulation to recover the received binary symbols. 


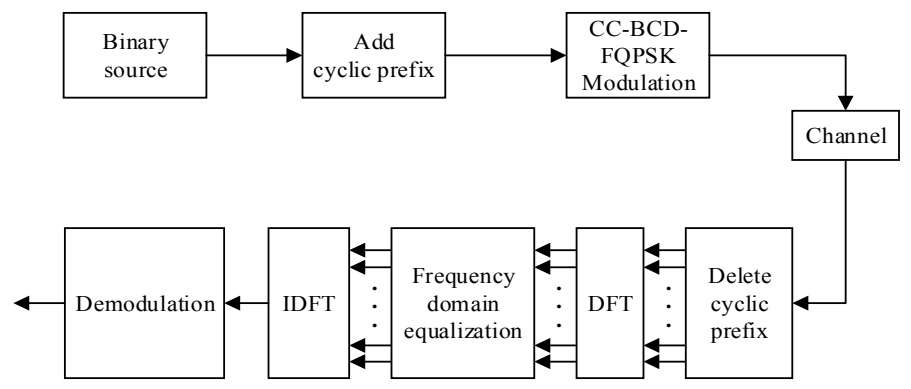

Fig.4. The basic structure of SC-FDE system based on CC-BCD-FQPSK modulation

\subsection{Frame structure Design}

For the purpose of the waveform continuity of the transmitted signal to obtain high spectral efficiency and eliminating ISI, a length- $G$ cyclic prefixis designed to be appended to the transmitted FQPSK signal, and $G$ equals or exceeds the maximum channel multipath delay.By removing the cyclic prefix at the receiver and detecting the signal in the frequency domain, the ISI will be removed.

Addition of the cyclic prefix is not straightforward forFQPSK signal, because waveform continuity mustalways be maintained.

Assume that FQPSK signal $s(t)$ is modulated by the binary sequence $\left\{x_{n}\right\}$, $n=0,1, \ldots, N-1$. Because the transmitted signal is composed of many data block, to maintain waveform continuity between two adjacent blocks when a cyclic prefix is added, it is essential that a cyclic prefix is added to the binary sequence firstly and then FQPSK modulation is implemented.This is done by simply copying $l_{t}$ tail symbols $x_{N-l_{t}+1}, x_{N-l_{t}+2}, \cdots, x_{N}$ and appending them to the beginning of $\left\{x_{n}\right\}$ firstly. The newly formed symbol sequence donated as $\left\{\bar{x}_{n}\right\}$, is

$$
\bar{x}_{n}=x_{(n)_{N}}, \quad n=-l_{t},-l_{t}+1, \ldots,-1,0,1, \ldots, N-1
$$

where $(n)_{N}$ is the residue of $n$ modulo- $N$. The length- $\left(N+l_{t}\right)$ sequence $\left\{\bar{x}_{n}\right\}$ is applied to FQPSK modulator to form the corresponding signal $\bar{s}(t)$.

Given that the oversample multiple is $p$, then modulated samples length of the binary sequence $\left\{x_{n}\right\}$ is $M=p \times N$, and $\bar{s}(t)$ samples length is $L=p \times\left(N+l_{t}\right)$. Because the FQPSK modulation is a memory-modulated method andthe first 4 symbols of the $\left\{\bar{x}_{n}\right\}$ are affected with the previous block, actual cyclic prefix length is $G=p \times\left(l_{t}-4\right)$, which must equal or exceed the maximum channel multipath delay. The illustration is shown as Figure 5.Though this method of cyclic prefix design has some redundant samples, it can maintain waveform continuity of each data block.

\begin{tabular}{|c|c|c|c|}
\hline \multicolumn{4}{|c|}{$\stackrel{G}{\longrightarrow}$} \\
\hline $\mathrm{CP}$ & Data & $\mathrm{CP}$ & Data \\
\hline
\end{tabular}

Fig. 5. Cyclic prefix design 
Given that the initial state of CC-BCD-FQPSK modulation of the first data block is 0 , record the final state, which is used to be the initial state of next data block, so that waveform continuity of the transmitted signal is maintained.It is worthily noted that initial state and final state of the binary source sequence $\left\{x_{n}\right\}$ are same, which leads thatthe convolutional coding is actually tail-biting convolutional coding (TBCC).

At the receiver, cyclic prefix is removed to eliminate ISI. After frequency domain equalization, data blocks are transferred back to time domain, Viterbi algorithm is suggested for demodulation.

\subsection{Frequency Domain Equalization}

Frequency domain equalization means that the equalization is done in frequency domain rather than in time domain. The basic concept is to compensate the frequency characteristics of the baseband system via the frequency characteristics of the tunable filter, so that the features of the baseband system including tunable filters can meet the requirements of no distortion transmission. As shown in Figure 4, the received signal is equalized in the frequency-domain after the DFT unit to estimate the channel parameters and make compensation of the frequency characteristics.

There are some common methods of frequency domain equalization, such as zeroforcing (ZF) equalization, minimum mean square error (MMSE) equalization and least squares (LS) equalization [6]. In this paper, MMSE equalization is applied to compensate the frequency characteristic, which can greatly reduce computation complexity compared to time domain equalization for channels having long impulse response.

\subsection{Demodulation}

The equalized signal is transferred back into the time domain by using IDFT to be demodulated. As described in section 3.1, the transmitted data blocks are actually modulated by TBCC. Frequency domain equalization and DFT/IDFT don't change the character of initial state same with final state of data block, so demodulating algorithms for TBCC can be used, which are mostly based on Viterbi Algorithm (VA) [7].

In this paper, wrap-around Viterbi algorithm (WAVA) is used to demodulate the received samples to recover the binary symbols, which is one of the circular Viterbi Algorithm (CVA) and uses the loopback characteristics of the convolutional code to repeat the received data blocks several times to form a long sequence for Viterbi decoding until the stop condition is satisfied.

\section{Simulation results}

As illustrated as figure 6, compared to linear modulation QPSK, the spectrum of FQPSK modulation is more compact and has less high frequency components and higher frequency band utilization. 


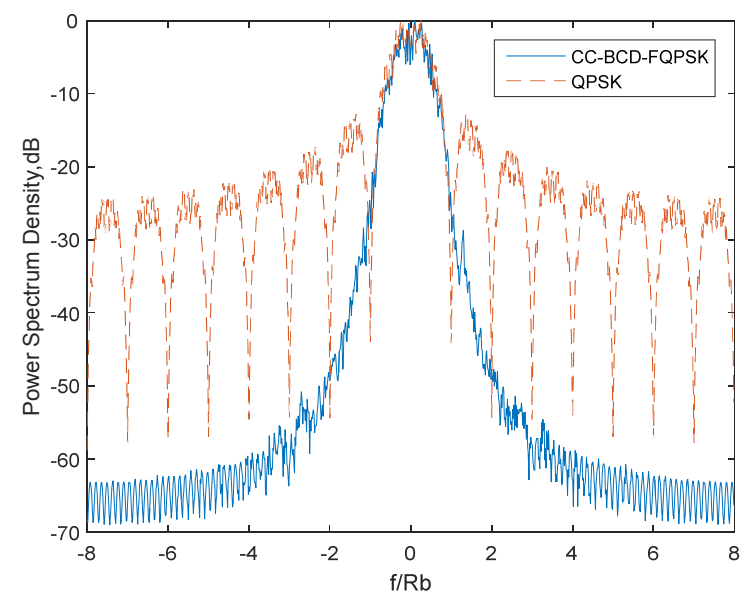

Fig.6. The power spectrum destiny of CC-BCD-FQPSK modulation and QPSK modulation

According to the structure of SC-FDE system based on CC-BCD-FQPSK modulation, simulation platform is built. Spatial channel model (SCM) is set as channel model. Binary data block length of I, Q paths is $N=1024$, tail symbols length is $l_{t}=80$, oversample multiple is $p=16$, so the actual cyclic prefix length is $G=(80-2) * 16=1248$. The modulated signals get through SCM channel and be recovered to binary symbols at the receiver. The bit error ratio (BER) performance is shown as Figure 7.

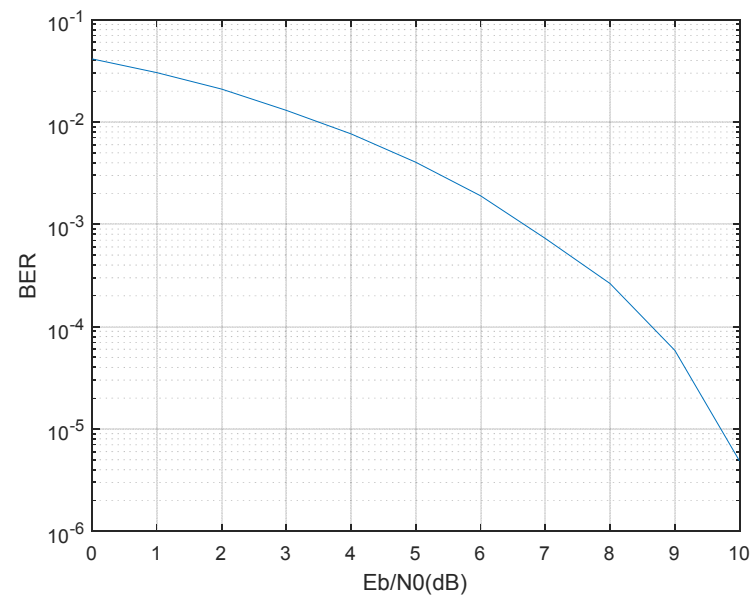

Fig. 7. BER performance of SC-FDE system based on CC-BCD-FQPSK modulation.

\section{Conclusions}

This paper considers applying FQPSK modulation for SC-FDE system. Firstly, this paper puts forward a new modulation method called CC-BCD-FQPSK modulation. To apply a DFT/IDFT on FQPSK signal, oversampling is used to generate a discrete representation for the FQPSK signal. Secondly, to maintain the waveform continuity of FQPSK signal, cyclic prefix is designed. Adding cyclic prefix first and then implementing FQPSK modulation is necessary for waveform continuity, which is different from implementing modulation first and then adding cyclic prefix of conventional SC-FDE. The simulation results demonstrate 
that on the basic of providing good BER performance, SC-FDE system based on CC-BCDFQPSK modulation attains excellent spectrum efficiency for multipath channel.

However, the work of this paper still has lots of deficiencies and defects, remaining to be further improved and perfected.

\section{References}

1. Feher, K. 1982. Filter: U.S. Patent 4339,724.

2. Kato, S. and Feher, K. 1986. Correlated signal processor: U.S. Patent 4567,602.

3. Leung, P. S. K. and Feher, K.1993.F-QPSK - A Superior Modulation Technique for Mobile and Personal Communications. IEEE Transactions on Broadcasting, 39(2): 288-294.

4. Kato, S. and Feher, K. 1983. XPSK: A New Cross-Correlated Phase-Shift Keying Modulation Technique. IEEE Transactions on Communications, 31: 701-707.

5. Mehdi, H. and Feher, K. 1994. FQPSK, power and spectral efficient family of modulations for wireless communication systems. Vehicular Technology Conference, 1994 IEEE 44th. IEEE: 1562-1566.

6. Simon, M. K. and Yan, T. Y. 1999. Performance Evaluation and Interpretation of Unfiltered Feher-Patented Quadrature Phase-Shift Keying (FQPSK). TMO Progress Report, 42-137.

7. Tan, J. and Stuber, G.L.2005. Frequency-domain equalization for continuous phase modulation. IEEE Transactions on Wireless Communications, 4(5): 2479-2490.

8. Nakao, M., Ishihara, T. and Sugiura, S. 2017. Single-Carrier Frequency-Domain Equalization with Index Modulation. IEEE Communication Letters, 21(2):298-301.

9. Pancaldi, F. and Vitetta, G. M. 2006. Equalization Algorithms in the Frequency Domain for Continuous Phase Modulations. IEEE Transactions on Communications, 54(4): 648-658.

10. Van, T. W., Nsenga, J., et al. 2008. Applying Frequency Domain Equalization to Precoded CPM. IEEE International Conference onAcoustics, Speech and Signal Processing (ICASSP): 2901-2904.

11. Thillo, W. V., Nsenga, J., et al. 2009. Low-complexity Frequency Domain Equalization for Continuous Phase Modulation. IEEE Transactions on Wireless Communications, 8(3):1435-1445.

12. Hassan, E. S., Zhu Xu, et al. 2009. A Continuous Phase Modulation Single-carrier Wireless System with Frequency Domain Equalization. IEEE International Conference on Computer Engineering and Systems.

13. Shao, R., Lin. S., Fossorier, M. P., et al. 2003. Two Decoding Algorithms for Tail biting Codes. IEEE Transactions on Communications, 51(10): 1658-1665.

14. Pai, H.R., Han, Y.S., et al. 2008. Low-Complexity ML Decoding for Convolutional Tail-Biting Codes. IEEE Communications Letters, 12(12): 883-885. 